\title{
The Effect of Verapamil on the Treatment of Detrusor Hyperreflexia in the Spinal Cord Injured Population
}

\author{
D. R. Bodner, MD, R. Lindan, MB, BS, E. Leffler, RN, M. I. Resnick, \\ MD \\ Division of Urology and Department of Orthopaedics, Case Western Reserve Univer- \\ sity School of Medicine and Spinal Cord Injury Units, Cleveland Metropolitan \\ General Highland View Hospital and Cleveland Veterans Administration Medical \\ Center, Cleveland, Ohio, USA.
}

\begin{abstract}
Summary
Verapamil, a calcium channel blocker, was tested alone and in combination with oxybutynin chloride for its clinical effectiveness in the treatment of detrusor hyperreflexia in a spinal cord injured population. Fourteen patients with detrusor hyperreflexia were included. All patients were treated with oxybutynin chloride alone and with the combination of oxybutynin chloride and verapamil. Six of the patients were also studied on no medication and verapamil alone. Cystometric and clinical comparisons were made with each change in drug dosage. Verapamil 240SL, when used alone, produced a delay in the first detrusor contraction as compared to no medication in 5 of the 6 patients but clinical improvement was insignificant. Clinical improvement with tolerable side effects was noted in 13 of the 14 patients treated with the combination of oxybutynin chloride and verapamil over oxybutynin alone. Our early experience with verapamil suggests that it may be a valuable adjunct in the treatment of detrusor hyperreflexia.
\end{abstract}

Key words: Spinal cord injury patients; Detrusor hyperreflexia; Calcium channel blockers; Anticholinergics; Verapamil.

Detrusor hyperreflexia is common in spinal cord injured patients when the level of injury is above the sacral reflex arc. Pharmacological control of reflex detrusor contractions is indicated in patients with chronic indwelling urethral catheters to prevent bladder trabeculation, urine leakage and catheter extrusion and is also indicated in some patients on an intermittent catheterisation program to prevent urinary incontinence between catheterisations. The drug most commonly used in the United States for this purpose is oxybutynin chloride. Like all the anticholinergic agents, oxybutynin causes side-effects at therapeutic levels. Dryness of the mouth is most frequently noted and at higher doses, this side-effect may be intolerable to many patients. Blurring of vision may also 
occur interfering with the patient's ability to drive, read or work. For these reasons, we have been interested in evaluating other agents to either replace or augment the actions of oxybutynin.

Terodiline, which is not yet available in the United States, has been reported by Scandinavian investigators to control motor urge incontinence in nonparalysed women by combining calcium antagonist, anticholinergic, sposmolytic, and local anesthetic properties. (Andersson, 1984; Henry, 1980; Klarskov et al., 1986; Lukkarinen et al., 1987). The calcium channel blocking agent verapamil, has been reported to have the ability to prevent detrusor contractions both in experimental animals in vitro and in vivo by interfering with the passage of calcium ions into smooth muscle cells (Andersson et al., 1986; Khanna et al., 1983; Maggi et al., 1982; Maggi et al., 1984). The primary use of this drug has been in the treatment of cardiac arrhythmias in humans. We were interested in testing the effect of verapamil alone and in combination with oxybutynin chloride, an anticholinergic, sposmolytic in the treatment of detrusor hyperreflexia. Our initial experience with verapamil for the treatment of detrusor hyperreflexia is reported herein.

\section{Patients and methods}

Fourteen patients followed up by the spinal cord injury units at Cleveland Metropolitan General Highland View Hospital and the Cleveland Veterans Administration Medical Centre with detrusor hyperreflexia who complained of intolerable side-effects from their dosage of oxybutynin choloride were entered in the study. There were 10 men and 4 women. Patients age ranged from 19 to 55 years with a mean of 34.2 years. Ten of the patients were on intermittent catheterisation and 4 had indwelling catheters. Eleven of the patients had urine leakage between intermittent catheterisations or leakage about the catheter that could not be managed with oxybutynin in dosages tolerable to the patient. Two additional patients were dry but complained of a severely dry mouth or blurred vision from the oxybutynin and were wet when the oxybutynin dosage was lowered. A final patient with detrusor-sphincter dyssynergia who had refused sphincterotomy had autonomic dysreflexia improved but not eliminated with oxybutynin and intermittent catheterisation. Twelve of the patients had spinal cord injury and 2 had neurological disease, one from spinocerebellar degeneration and the other from multiple sclerosis. There were 5 cervical injuries (3 complete and 2 incomplete) and 7 thoracic injuries of which 6 were complete. The mechanism of injury consisted of 5 motor vehicle accidents, 4 falls, 1 diving accident, and 2 transverse myelitis. Patients were entered in the study from 5 months to 27 years (mean of 7.6 years) after spinal cord injury or diagnosis of the neurological disease. Informed consent was obtained from each patient. Urine cultures were obtained and the urine was sterilised with appropriate antibiotics based upon sensitivity studies prior to starting a patient on the study. Baseline water cystometrics were obtained on a DISA Dantec 2100 urovideo urodynamics unit and true bladder pressures were determined after subtracting rectal pressures. Urine cultures, liver function tests, and EKGs were obtained as baseline studies and repeated along with cystometrics and 3 to 7 days after 
Table Effect of verapamil on bladder volume at first detrusor contraction ( $\mathrm{ml}$ water)

\begin{tabular}{|c|c|c|c|c|}
\hline Patient & No meds & Verapamil alone & Oxy only & Oxy/verap \\
\hline 1 & 20 & 70 & 220 & $260^{\star}$ \\
\hline 2 & 20 & 80 & 150 & 190 \\
\hline 3 & 75 & 140 & 250 & $250^{\star}$ \\
\hline 4 & 80 & 188 & 270 & 330 \\
\hline 5 & 320 & 280 & 400 & 600 \\
\hline 6 & 160 & 200 & 250 & 280 \\
\hline 7 & & & 100 & 160 \\
\hline 8 & & & 150 & $370^{\star}$ \\
\hline 9 & & & 160 & $290 \star$ \\
\hline 10 & & & 30 & 60 \\
\hline 11 & & & 60 & 250 \\
\hline 12 & & & 150 & $235^{\star}$ \\
\hline 13 & & & 280 & 500 \\
\hline 14 & & & 120 & $250^{\star}$ \\
\hline
\end{tabular}

\#Patient dry and free of the leg bag on the combination of verap/oxy but was incontinent with oxy alone

$\star$ Patients with lower dosage of oxybutynin when the combination of oxy/verap was used over oxy alone.

each change in drug dosage. At least two different cystometrics were obtained on each drug dosage.

All patients were placed initially on oxybutynin. Eleven were on $5 \mathrm{mg} \mathrm{q} 6$ hours, 2 were on $5 \mathrm{mg} \mathrm{q} 8$ hours, and one was on $10 \mathrm{mg} \mathrm{q} 6$ hours with imiprimine $50 \mathrm{mg}$ hs. Six patients were taken off all medications for 3 to 7 days and baseline CMGs were obtained. These were then compared with those obtained while treating the patient with verapamil 240 SL q am alone. Because of severe detrusor hyperreflexia, oxybutynin was not discontinued in the other 8 patients. Oxybutynin dosages were titrated from $2.5 \mathrm{mg}$ q 8 hours to 5 mg q 6 hours until the patient complained that the side-effects were unpleasant. Verapamil $240 \mathrm{SL}$ was then added in the morning and if the patient was not dry with this combination an additional verapamil 120 SL was added at bedtime. During the titration period, patients were kept on each drug dosage for a minimum of 3 to 7 days. Once the final drug dosage was determined, patients were followed on a monthly basis with liver functions repeated every 3 months. The average follow-up was 6 months.

\section{Results}

The effect that verapamil had on the first detrusor contraction is shown in the Table. Delay in the first detrusor contraction was noted in 5 of the 6 patients treated with verapamil $240 \mathrm{SL}$ alone as compared to the same patient on no medications. However, these patients continued to have uninhibited bladder contractions at relatively low bladder volumes and clinical improvement was not noticeable with verapamil alone. Thirteen of the 14 patients were clinically improved with the combination of oxybutynin with verapamil over oxybutynin alone. They were significantly more dry clinically, with minimal leaking in-between intermittent catheterisations permitting elimination of the leg bag in the male patients on intermittent catheterisation and minimal leaking about the indwelling catheter. Nine of these patients remained on the same 


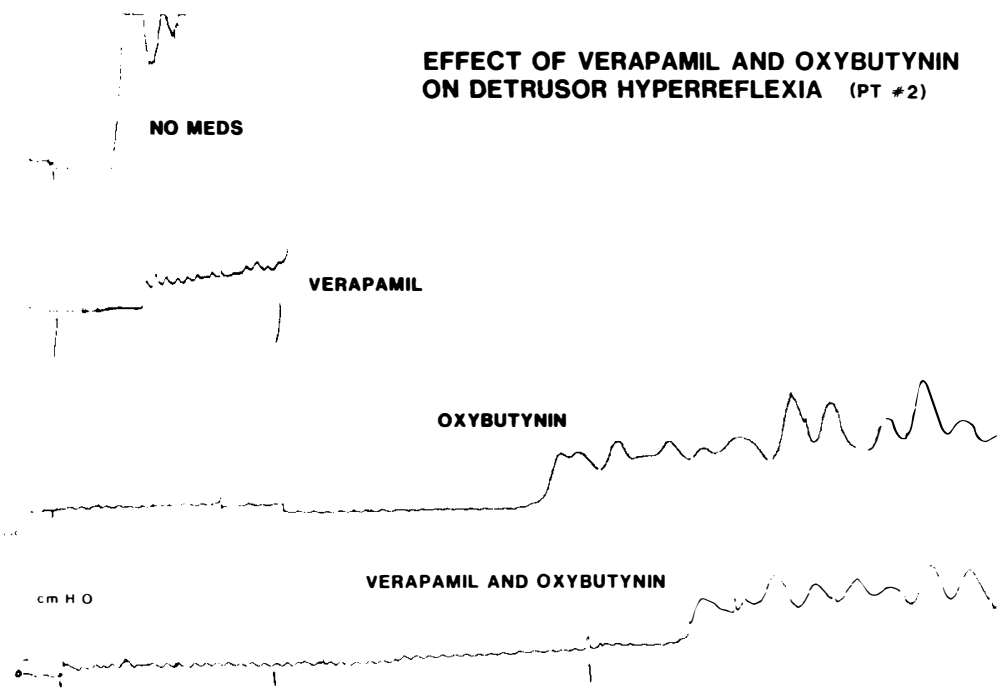

Figure 1 Comparison of cystometric findings with various medications in spinal cord injured patients with detrusor hyperreflexia (patient $\$ 2$, Table 1 )

Note the improvement in bladder capacity and decrease in intravesical pressure with verapamil over no medications, oxybutynin over verapamil alone, and oxybutynin and verapamil over oxybutynin alone.

oxybutynin dosages as they were on prior to the study but 8 of the 9 became clinically drier without increasing the oxybutynin dosage. It was possible to lower the dosage of oxybutynin in the remaining 5 patients with the addition of verapamil and these patients noted that they were clinically more dry than they had been on the higher dosages of oxybutynin alone. Final drug dosages consisted of oxybutynin $5 \mathrm{mg} \mathrm{q} 6$ hours with verapamil 240 SL in 8 of the patients, oxybutynin $5 \mathrm{mg}$ q 8 hours with verapamil $240 \mathrm{SL}$ q am and verapamil $120 \mathrm{SL}$ q hs in 2, oxybutynin $5 \mathrm{mg}$ q 8 hours with verapamil 240 SL in 2 and oxybutynin $2.5 \mathrm{mg} \mathrm{q} 8$ hours with verapamil $240 \mathrm{SL}$ in 2 . The one patient that showed no clinical improvement from the combination of oxybutynin and verapamil had a small fibrotic bladder with a capacity less than $50 \mathrm{cc}$. The effect of the combination of verapamil and oxybutynin on the first detrusor contraction is shown in the Table. The volume at which the first detrusor contraction occurred was increased in 12 of the patients on the combination of oxybutynin with verapamil. Figure 1 reveals the cystometric findings with various medications used in patient $\$ 2$ (described in the Table). Figure 2 reveals improvement in urodynamic findings in a patient leaking about his catheter when verapamil was added to oxybutynin (patient $\$ 7$ ). Three patients had autonomic dysreflexia elicited with each cystometric examination. The maximal systolic blood pressure was reduced by 20 to $30 \mathrm{~mm}$ $\mathrm{Hg}$ consistently in each of these patients once verapamil was added to their treatment regimen.

Eleven of the patients complained of a mild increase in constipation shortly after starting verapamil, but this was self-limited in all the patients. No changes were noted in the serial EKGs or liver function tests. 


\section{DRUG EFFECT ON HYPERREFLEXIA}

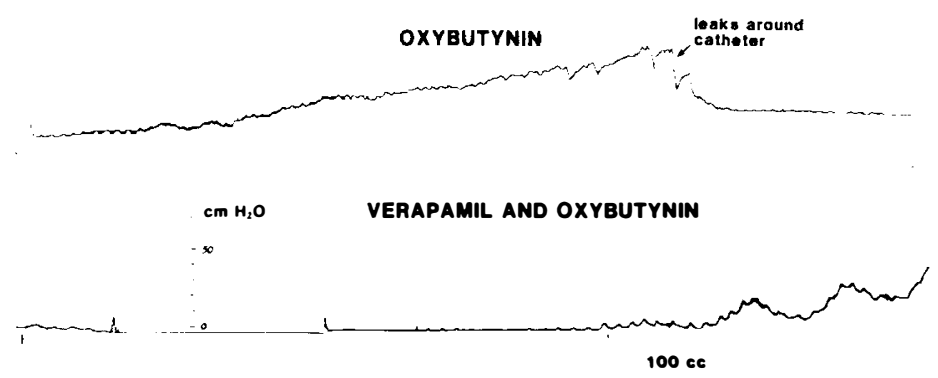

Figure 2 This quadriplegic patient had a chronic indwelling catheter with urine leaking about the catheter despite oxybutynin therapy. The bladder capacity increased and incontinence about the catheter decreased with the addition of verapamil.

\section{Discussion}

Uninhibited detrusor contractions remain a problem for the spinal cord injured patient with lesions above the sacral reflex arc. Treatment is indicated in patients on intermittent catheterisation to keep them dry between catheterisations and in patients with indwelling catheters to prevent leakage about the catheter and prevent the development of bladder trabeculation or catheter extrusion. Pharmacological treatment in the US has for many years been the anticholinergic drug oxybutynin chloride. While this medication is clinically effective, many patients become non-compliant in taking the drug because of the undesirable side-effects.

Calcium entry from an extracellular medium appears to play an important role in the activation of urinary bladder smooth muscle (Forman et al., 1978). Drugs that block the influx of calcium should therefore be expected to have some inhibitory effect on bladder smooth muscle contraction. The combination of an anticholinergic with a calcium blocking agent offers the potential pharmacotherapy advantage of increasing the therapeutic effectiveness while decreasing the side-effects encountered with adequate dosages of anticholinergics (Andersson and Ulmsten, 1980, Andersson, 1984). Terodiline, a drug produced by the Kabi-Company (Sweden), an agent which combines anticholinergic, calcium channel blocking, sposmolytic and local anesthetic properties has been reported to be more effective than placebo in the treatment of urge incontinence in non-paralysed women (Klarskov et al., 1986; Lukkarinen et al., 1987). As this agent is not available in the United States, verapamil, a calcium channel blocker, that had been reported to block detrusor smooth muscle in vivo and in vitro (Henry, 1980; Khanna et al., 1983) was chosen to be tested alone and in a combination with oxybutynin chloride.

From our initial experience, it appears that verapamil does delay the first detrusor contraction; however, when used alone, the efféct was insufficient to be of any significant clinical value. The combination of verapamil and oxybutynin produced clinical improvement over oxybutynin alone in 13 of the 14 patients. The patient that showed no benefit had a small fibrotic bladder and was perhaps a poor candidate for this study and may require 
bladder augmentation. Side-effects of therapeutic dosages of the combination of verapamil with oxybutynin were better tolerated than clinically effective dosages of oxybutynin alone and consisted only of transient constipation. Dryness of mouth was improved.

Verapamil also appeared to blunt the hypertensive response elicited by cystometrics in the 3 patients that had developed autonomic dysreflexia by this stimulus. This added effect of verapamil may make its utilisation in this patient population more desirable.

\section{Conclusion}

While this is an initial study on a limited number of patients, it would appear that verapamil is of benefit when used in combination with oxybutynin in the treatment of detrusor hyperreflexia. Side-effects with this combination were more tolerable than with oxybutynin alone. An added benefit appeared to be a blunting of autonomic dysreflexia once verapamil was begun. Further studies combining calcium channel blockers and anticholinergics appear warranted in the treatment of detrusor hyperreflexia. The combination may minimise side effects of the medications and improve patient compliance.

\section{References}

ANDERSSON K-E 1984 Clinical Pharmacology of Terodiline. Scandinavian fournal of Urology and Nephrology. Supplementum 87:13-20.

ANDERSSON K-E, FORMAN A 1986 Effects of calcium channel blockers on urinary tract smooth muscle. Acta Pharmacologica et Toxicologica 58(Suppl. 2):193-200.

ANDERSSON K-E, Ulmsten U 1980 Drug treatment of the overactive detrusor. Acta Pharmacologica et Toxicologica 46(Suppl I:7.

Forman A, ANDERSSON K-E, HeNriksson L et al. 1978 Effects of nifedipine on the smooth muscle of human urinary tract in vitro and in vivo. Acta Pharmacologica et Toxicologica (Kbh,43:111.

HENRY PD1980 Comparative pharmacology of calcium antagonists: nifedipine, verapamil and diltiazem. American fournal of Cardiology 46:1047-1058.

KhANNA P, BARBIERI EJ, Moss M et al. 1983 Effects of calcium and verapamil on vesicourethral smooth muscle of rabbits. Urology 21:284-290.

KLARSKOV P, GERSTENBERG TC, HALD T 1986 Bladder training and Terodiline in females with urge incontinence and stable detrusor function. Scandinavian fournal of Urology and Nephrology 20:41-46.

LuKKarinen O, Grohn P, Wilen-Rosenqvist G et al. 1987 A controlled, double-blind, crossover study of terodiline in motor urge incontinence. Annales Chirurgiae et Gynaecologiae 76:128-132.

Maggi CA, Grimaldi G, Meli A 1982 The effect of nifedipine and verapamil on spontaneous and carbachol induced contractions of rat urinary bladder in vivo. Arch. Int. Pharmacodyn. Ther. 258:288-294.

MAGgi CA, MELI A 1984 The effect of nifedipine and verapamil on $\mathrm{KCl}$-induced rhythmic contractions of guinea pig ureter in vitro. Experientia, 40:681-686. 